\title{
Optimal Clustering in Wireless Sensor Networks for the Internet of Things Based on Memetic Algorithm: memeWSN
}

\author{
Masood Ahmad $\left(\mathbb{D},{ }^{1}\right.$ Babar Shah $\left(\mathbb{D},{ }^{2}\right.$ Abrar Ullah, ${ }^{3}$ Fernando Moreira $\left(\mathbb{D},{ }^{4}\right.$ Omar Alfandi $(\mathbb{D})^{2}$ \\ Gohar Ali $(1)^{5}$ and Abdul Hameed $\oplus^{6}$ \\ ${ }^{1}$ Department of Computer Science, Abdul Wali Khan University, Pakistan \\ ${ }^{2}$ College of Technological Innovation, Zayed University, Abu Dhabi, UAE \\ ${ }^{3}$ Heriot-Watt University, UK \\ ${ }^{4}$ Head of Science and Technology Department, Universidade Portucalense, Portugal \\ ${ }^{5}$ Department of Information Systems and Technology, Sur University College, Oman \\ ${ }^{6}$ Department of Computing and Technology, Iqra University, Islamabad, Pakistan \\ Correspondence should be addressed to Babar Shah; babar.shah@zu.ac.ae
}

Received 3 July 2020; Revised 2 December 2020; Accepted 16 December 2020; Published 6 January 2021

Academic Editor: Ki-Il Kim

Copyright (C) 2021 Masood Ahmad et al. This is an open access article distributed under the Creative Commons Attribution License, which permits unrestricted use, distribution, and reproduction in any medium, provided the original work is properly cited.

\begin{abstract}
In wireless sensor networks for the Internet of Things (WSN-IoT), the topology deviates very frequently because of the node mobility. The topology maintenance overhead is high in flat-based WSN-IoTs. WSN clustering is suggested to not only reduce the message overhead in WSN-IoT but also control the congestion and easy topology repairs. The partition of wireless mobile nodes (WMNs) into clusters is a multiobjective optimization problem in large-size WSN. Different evolutionary algorithms (EAs) are applied to divide the WSN-IoT into clusters but suffer from early convergence. In this paper, we propose WSN clustering based on the memetic algorithm (MemA) to decrease the probability of early convergence by utilizing local exploration techniques. Optimum clusters in WSN-IoT can be obtained using MemA to dynamically balance the load among clusters. The objective of this research is to find a cluster head set (CH-set) as early as possible once needed. The WMNs with high weight value are selected in lieu of new inhabitants in the subsequent generation. A crossover mechanism is applied to produce new-fangled chromosomes as soon as the two maternities have been nominated. The local search procedure is initiated to enhance the worth of individuals. The suggested method is matched with state-of-the-art methods like MobAC (Singh and Lohani, 2019), EPSO-C (Pathak, 2020), and PBC-CP (Vimalarani, et al. 2016). The proposed technique outperforms the state of the art clustering methods regarding control messages overhead, cluster count, reaffiliation rate, and cluster lifetime.
\end{abstract}

\section{Introduction}

Wireless sensor network-enabled Internet of Things (WSNIoT) is the set of WMNs capable to share data with their neighbors. WSN-IoT can be used for managing different applications such as rescue, flood monitoring, monitoring a border between two states, managing a disaster, and communication in the battlefield among others. The clustering techniques commence awesome once the size of WSN-IoT turns into a massive network in comparison to flat WSN irrespective of routing structure implemented [1]. The scalability problem in flat WSN is a serious concern when a big total of mobile knots are moving in different directions. When the number of WMNs in WSN-IoT using a flat routing arrangement is $x$, then the complexity of proactive routing structure will be $O\left(x^{2}\right)$ [2]. While the number of WMNs in WSN-IoT grows, the routing overhead also increases with the ratio of the square the number of WMNs. The reactive routing algorithms similarly result in route setup delay once the number of WMNs in a WSN-IoT increases. Furthermore, the flooding route request packets fear may similarly rise which further slowdowns the network. Hence, to accomplish elementary performance assurance in sized WSN-IoT, a hierarchal structure is compulsory [3]. The common execution of a hierarchal strategy is the clustering organization. Cluster formation is a challenging task in designing the cluster- 
based routing schemes since the choice of optimal $\mathrm{CH}$-set is an NP-hard problem [4].

Planning a clustering structure to route QoS information is the main requirement of the WSN-IoT study. Clustering is the main prototype, and its significance can be listed in two ways. Primarily, WSN-IoT management can be accomplished meritoriously thru clustering structure. An ordinary WSN-IoT contains above a hundred or even a thousand WMNs. In flat WSN-IoT configuration, unneeded packets are transported from source to sink nodes [5]. The scalability problem may possibly rise with flat-based WSN when we want to increase the number of WMNs in WSN and may saturate the network. The WMNs in WSN-IoT may move, and controlling the scalability is extra stimulating in contrast to static networks. Hence, cluster-based routing can be used for the effective management of WSN-IoT. Moreover, clustering will help answer queries such as topology control, building a virtual network, and intrusion discovery [6].

One of the arrogant design concerns of a cluster-based routing algorithm is finding an ideal $\mathrm{CH}$-set that is supposed to shelter the whole WSN-IoT network area. Each time a $\mathrm{WMN}$ is associated with a cluster, however, it is not compulsory that all clusters have a $\mathrm{CH}$. Since the presence of a $\mathrm{CH}$ in a cluster has the advantage of managing the WSN-IoT easily, the majority of the existing work assumes the presence of $\mathrm{CHs}$ in each cluster. The construction of clusters may be performed in a manner that should reduce the message overhead that occurred during the cluster formation phase. If not, the proposed cluster-based routing scheme will consume more energy as compared to the flat routing protocol. Discovering optimal $\mathrm{CH}$-set may be carried out using optimization algorithms, i.e., evolutionary genetic algorithms (GA), neural networks, and particle swarm optimization (PSO) [7]. The particle swarm optimization (PSO) scheme is not suitable because PSO falls easily to optima when the space is high dimensional. PSO also has a low convergence rate in the iterative process. The bee colony optimization lacks the use of secondary information. It also needs novel fitness checks on new algorithm parameters. A higher number of objective function evaluation is essential in BCO. The algorithm may perform slowly in serial processing. The genetic algorithm is very slow and is not suitable for WSN where the resources are limited.

In this article, the WSN-IoT is disseminated to multiple subgroups known as clusters with an evolutionary MemA. The research question of the clustering issue is assumed as a graph plus the suitability model is verified as per the need of WSN-IoT. In the suggested memeWSN, the algorithm chooses a $\mathrm{CH}$-set. The $\mathrm{CH}$-set indicates a chromosome (optimum elucidation). The worth of the optimal solution is enhanced using a confined search technique. Moreover, the $\mathrm{CH}$-set outcome is estimated using a fitness function (FF). The paternities nominated are intended for the replica on the basis of fitness value (FV). Mutation in addition to crossover can be used to bring diversity in the population and to generate different offsprings. The new-fangled chromosomes are produced as well as verified till the ideal $\mathrm{CH}$-set originate. The effectiveness of the clustering procedure is improved using MemA as exposed in simulation results. The experi- mental results prove that the advised technique devises prominent performance while matching with state of the art clustering schemes.

The remaining article is structured as follows: in Section 2 , we argue the relevant research presented in recent years. Section 3 presents our proposed system designed on the basis of MemA. In Section 4, the formulation of WSN-IoT using MemA is presented. In Section 5, we conclude the article, and the future research directions are stated.

\section{Literature Review}

The main goal of the proposed method is to reduce the energy depletion during the design of the cluster. In [8], a clustering procedure has been proposed which works in a distributed way termed distributed $\mathrm{CH}$ scheduling (DCHS) algorithm to enhance the lifetime of WSN. The key parameter of node mobility and a large number of neighbors are not considered in the scheme as well as in the selection of $\mathrm{CH}$. Furthermore, the important parameters such as communication load, reputation, and trust are passed over as well in the paper. The authors in [9] selected CHs randomly in the first round where the criteria in the second round for $\mathrm{CH}$ selection is based on residual energy. However, the result of the arbitrary choice of $\mathrm{CH}$-set in the $1^{\text {st }}$ phase causes a derange partition. The movement and degree of MNs for the duration of the $\mathrm{CH}$-set selection is also snubbed.

The mobility of sensor nodes is key to consider during the selection of CHs to increase the lifetime of WSN-IoT. The node motion may be in random directions or may practice a different movement configuration. In leader-based group routing, the WMNs are assembled into a group under the umbrella of a leader [10]. Each group in the network assumes a different element to reduce the requests initiated for different resources. In this way, the throughput will increase, and routing overhead will reduce. The separate consignment of resources to each group is made to minimize the influence of group dissimilarities on the efficiency of WSNIoT. In [11], the mobility of WMNs is considered major criteria to select the CHs. The distinguished feature of a WMN is its lower mobility and most suitable node to perform the $\mathrm{CH}$ role. A weighted cluster-based scheme is assumed to accomplish the determination of $\mathrm{CHs}$ and the association of their member nodes. The WMNs with minimum motion are the top contestants for the $\mathrm{CH}$ role. The proposed algorithm may not execute fine while the nodes in WSN are moving with fast speed. The WMNs with little speed will detach eventually, and the cluster formation method will initiate repeatedly which will decrease the lifetime of WSN. Correspondingly, the low mobility WMN selected as $\mathrm{CH}$ on the other hand with a dissimilar track than its neighbors may result in connectivity interruption to their sisters. The $\mathrm{CHs}$ are selected without considering the node degrees. We suggest consideration of some or all parameters such as residual energy, node neighbors, and comparative mobility during the cluster formation.

The mobility of WMNs is predicted accurately to handle the topology changes due to the high mobility proposed in [12]. The scheme forms a cluster and maintains it based on 
predicting the future mobility of nodes instead of a fixed geographical partition. In contrast to other WMNs in MANET, the WMNs with low speed is the ideal candidate for the cluster head role. The movement pattern of long time neighbor WMNs is used to calculate the movement ratio of a WMN. The WMNs with high probability demonstrate that the $\mathrm{WMN}$ is moving alongside its neighbors at equal speed and pattern or is moving with slight motion. It illustrates that a WMN will assist its neighbors for an extended duration and is a better nominee to become a cluster head. The objective of the scheme is as follows: due to mobility, the clusters that are highly resistant to topological changes are formed with the right prediction of the WMN mobility. The prediction of mobility is made on the basis of the WMN location relative to their neighbors. The location is checked over different intervals, and therefore, no special hardware is required for the purpose. The accurate calculation is possible if we consider and check the correlation of WMNs moving in the vicinity. The WMNs with relative mobility and high degree are ideal candidates to form stable clusters. The performance of the protocol may degrade when the mobility model is random and may increase control messages overhead. It is difficult to predict the future mobility of WMNs in many scenarios. Neighbor's quality is ignored. Local changes in topology may initiate the reclustering procedure but due to dynamic topology, the WMNs may not join the same cluster in the next round.

In the dynamic genetic algorithm-based clustering, the load balance issue is initially modeled to a dynamic optimization problem [13]. Using various diverse dynamic GAs established for dynamic optimization is suggested to resolve the problem of balance cluster formation in a network. The capability of a $\mathrm{CH}$ set is evaluated on the load balance matrix, and each individual represents a clustering structure. To assist the population deal with variations in topology plus recommend closely related better solutions, several approaches are suggested. To handle the ecological dynamics, some multipopulation approaches, memory, immigrants, and the combination of all these are combined into SGA. The authors talk over several structures to tackle DLBP; however, it is not clear in the paper how the proposed schemes will be applied. The pseudocode stated in the paper is very basic. The proposal assumes the degree of a node to become a cluster head, and the performance may be unsatisfactory in several setups. If the WMNs are assigned the cluster head role on the basis of high mobility, it may perform poorly than flat routing protocols. The reclustering is initiated when the topology changes; it results worst if the topology does not change because the battery of the cluster head will quickly drain.

The authors of [14] proposed an optimization algorithm which finds the optimal clusters in multiobjective fashion to efficiently manage the resources of the network. With optimal clusters in MANET, the energy efficiency will increase by efficiently organizing the resources and the $\mathrm{CHs}$ are assigned the task of intracluster and intercluster communication. As an alternative to allocating a weight value to all parameters, it deals straight with the DOP so as to find the Pareto optimal solutions. Less cluster heads will reduce the hop count besides packet delay in a cluster-based routing protocol. By minimizing the number of clusters, we can reduce the routing cost of a packet. The degree of WMNs is not taken into consideration during the cluster head selection process. The ideal candidates for the role of $\mathrm{CHs}$ are nodes having high broadcast power, and the $\mathrm{CH}$ s may be selected from one part of the network. In this scenario, the energy consumed on cluster formation will be wasted. The precomputation of WMN energy dissipation may not be practical; this parameter may be considered as the remaining energy of a node. The key parameters, i.e., node speed and mobility model, are not measured in the simulation.

Mobility Aware Energy Efficient Clustering for Wireless Sensor Network (MobAC) is presented in [15]. The paper suggests a cluster-based routing algorithm to enhance the lifetime of WSN. The node distance, its lingering vitality, and mobility are assumed during the $\mathrm{CH}$ selection process. To further reduce the energy consumption, the paths that are energy-efficient, stable, and short are selected for packet forwarding. The degree and residual energy of nodes is not considered during the $\mathrm{CH}$ selection process. The $\mathrm{CH}$ may be selected from one part of the network, and the reaffiliation rate may increase.

A Proficient Bee Colony-Clustering Protocol to Prolong Lifetime of Wireless Sensor Networks (PBC-CP) is discussed in [16]. The nodes selected as CHs have more burden as compared to ordinary nodes in WSN. The CHs are selected based on the distance of a node from the base station, its degree, and energy. The energy-efficient paths are used to transmit data to other clusters and base stations. The mobility metric plays an important role in the selection of CHs. The PBC$\mathrm{CP}$ ignores the mobility of nodes during the $\mathrm{CH}$ selection. The nodes selected as CHs with different mobility than their neighbors may cause reclustering more frequently. Calling the reclustering procedure more repeatedly may result in unstable clusters, and the network lifetime may decrease.

An Enhanced PSO-Based Clustering Energy Optimization Algorithm for Wireless Sensor Network (EPSO-C) uses particle swarm optimization to select the $\mathrm{CH}$ and minimize the energy consumption [17]. In this approach, the particles are optimized to get the most optimal $\mathrm{CHs}$. The $\mathrm{CH}$ s are selected based on their location, and the $\mathrm{CH}$-set will cover the whole network. The location of nodes is used to select the CHs in WSN. The static nodes are assumed, but in the current and future networks, most of the nodes will be mobile or even the nodes will fly. The suggested method may fail if the nodes deployed in the network move from one location to another. The node degree and energy are also ignored in this scheme.

The strict nature of WMNs in WSN w.r.t energy and communication range make it challenging to communicate the identifying information on time with minimum delay. The routing problems in addition to small network time may also rise because of the WMN limited energy. The WMNs near the base station carry a substantial weight of information transmitted on behalf of other WMNs located nearby. The main goal of this proposal is to select the CHs from optimal sites, and the gateway WMNs will be chosen from all clusters based on their position. The presence of 
gateway nodes will reduce the burden on $\mathrm{CH}$. The gateway WMNs are responsible to transmit information intercluster and intracluster. This way, the load on the CHs will be balanced. The exposure of $\mathrm{CH}$ s will rise since the gateway WMNs transmit information with adjacent clusters on behalf of $\mathrm{CHs}$.

To overcome the issues found in the literature, the $\mathrm{CH}$ in WSN-IoT are selected based on multiple parameters such as WMN current energy, degree, and mobility. The current energy consideration increases the cluster lifetime. The balanced clusters are obtained by considering the WMN degree. To reduce the riffle effect of reclustering, the mobility of WMNs is considered during the cluster formation. The MemA is also applied to obtain the optimal CHs. The issue of premature convergence will not arise due to the local search technique embedded in the MemA.

\section{WSN-IoT Clustering Problem Formulation}

To model the matter of allocating the WSN-IoT into clusters, $k$ clusters and $N$ number of WMNs in WSN-IoT are assumed. In the proposed memeWSN, the model is first demonstrated followed by the formulation of the dynamic and optimum clustering in WSN-IoT. The difficulty of stable cluster development of $n$ WMNs is essentially resolved by discovering a set of WMNs (CH-set) which shows the division of $n$ WMNs into $k$ nonoverlapping clusters ( $\mathrm{C} 1$, $\mathrm{C} 2, \cdots, \mathrm{Ck})$. This can be achieved by modeling the WSNIoT as a graph $G(V, E)$, where $V$ represents the vertices of mobile nodes and $E$ represents the edge of the communication links between the WMNs in the graph.

A set of vertices (CHs) is selected optimally based on the $\mathrm{FV}$ of WMNs for the $\mathrm{CH}$ sole. The $\mathrm{CH}$ set with higher weighting values which covers most part of the WSN-IoT has a higher fitness value.

The following equation (1) calculates the weighting factor (WF) value of a WMN $i$.

$$
W_{\text {node }_{i}}=W E n r_{\text {Node }_{i}}+W N g h_{\text {Node }_{i}}+W M o b_{\text {Node }_{i}},
$$

Here, $W E n r_{\text {Node }_{i}}$ is the WF concerning the residual power of a WMN $i$ calculated as:

$$
\mathrm{AE}_{\mathrm{Node}}=\frac{\sum_{i=1}^{n} R \cdot E n r_{\mathrm{Node}_{i}}}{n}
$$

R.Enr Node $_{i}$ is the remaining power of a WMN $i, n$ is the total WMNs in WSN-IoT, plus $\mathrm{AE}_{\text {Node }}$ is the average power of WMNs at present time. The WF with regard to energy may be extracted as:

$$
\begin{aligned}
\text { if } R \cdot E n r_{\text {Node }_{i}} & >\mathrm{AE}_{\mathrm{Node}_{\mathrm{e}}} \text { then } \\
W E n r_{\mathrm{Node}_{i}} & =1 \\
\text { else if } R \cdot E n r_{\mathrm{Node}_{i}} & \approx \mathrm{AE}_{\mathrm{Node}} \text { then } \\
W E n r_{\mathrm{Node}_{i}} & =0
\end{aligned}
$$

$$
\begin{aligned}
& \text { else } \\
& W E n r_{\text {Node }_{i}}=-1
\end{aligned}
$$

The WF regarding energy will be 0 if the remaining energy of WMN "R.Enr $r_{\text {Node }}$ is nearly equal to the average energy $(\approx)$ "AE $\mathrm{ASN}_{\mathrm{WSOT}}$ " of WSN-IoT. WNgh Node $_{i}$ represents the weight factor with respect to neighbors of a WMN. The weight value $W N g h_{\text {Node }_{i}}$ can be assigned based on the inner and outer degree of WMNs for instance:

$$
A N g h_{\text {Node }}=\frac{\sum_{i=1}^{n}(\text { OuterDeg }+ \text { InnerDeg })_{\text {Node }_{i}}}{n},
$$

where OuterDeg and InnerDeg are the inner and outer degree of WMN $i, A N g h_{\text {Node }}$ is the typical neighbors in WSN-IoT. The WF w.r.t WMN neighbors can be allocated in the subsequent para as:

$$
\begin{aligned}
& \text { if }(\text { OuterDeg + InnerDeg })_{\text {Node }_{i}}>A N g h_{\text {Node }}>\text { then } \\
& W N g h_{\text {Node }_{i}}=1 \\
& \text { else if(OuterDeg }+ \text { InnerDeg })_{\text {Node }_{i}} \approx A N g h_{\text {Node }} \text { then } \\
& W N g h_{\text {Node }_{i}}=0 \\
& \text { else } \\
& W N g h_{\text {Node }_{i}}=-1
\end{aligned}
$$

Similarly, the WF of WMN regarding mobility $W M o b_{\text {Node }_{i}}$ can be computed as follows keeping in mind that WMN is having relative movements; otherwise, the static WMNs are the best nominees for the role of $\mathrm{CH}$.

$$
\begin{aligned}
& \text { if }(\text { mobility } \approx \text { relative or static }) \text { then } \\
& W M o b_{\text {Node }_{i}}=1 \\
& \text { else } \\
& W M o b_{\text {Node }_{i}}=-1
\end{aligned}
$$

$k$ is the number of clusters would be calculated before selecting the $\mathrm{CH}$-set through the equivalence in equation (7) for calculating the value of $k$ :

$$
k=\left\lceil\frac{\sum_{i=1}^{n}(\text { OuterDeg }+ \text { InnerDeg })_{\text {Node }_{i}}}{n}\right\rceil+1 .
$$

In the above equation (7), the total cluster in WSN-IoT is $k$, the total WMNs in WSN-IoT is represented by $n$, and the (OuterDeg + InnerDeg) Node $_{i}$ represents the neighbor information of node $i$.

The cluster head set covers WMNs as a minimum 3-hop away from another cluster head. Once the WFs of the whole WSN-IoT WMNs are calculated, the equation designated in equation (8) will be used to calculate the correctness of the 
CH-set.

$$
\begin{gathered}
\text { Minimize Fun }(W, \mathrm{AFV})=\sum_{i=1}^{n} \sum_{j=1}^{k} R \mathrm{Weight}_{i j}\left(W_{\text {node }_{i}}-\mathrm{AFV}_{\text {node }_{j}}\right)^{2}, \\
\text { Subject to } \sum_{j=1}^{k}\left(R \text { Weight }_{\text {node }_{j}}=1\right)=k \text { for }(j=1, \cdots, k), \\
R \text { Weight }_{\text {node }_{j}}=1 \text { or } 0, \text { for }(i=1, \cdots, n \text { and } j=1, \cdots, k) .
\end{gathered}
$$

The function stated using equation (8) is a minimization function where $n$ is the total WMNs in WSN-IoT, $k$ number of known or unknown (the number of clusters may not be known in advance) clusters will be designed, $W_{\text {node }_{i}}$ $(i=1,2,3, \cdots, n)$ is the weight of node ${ }_{i}$, and $\mathrm{AFV}_{j}$ is the average fitness value of $\mathrm{WMN}$ to accomplish the $\mathrm{CH}$ role. Equation (9) below will compute the $\mathrm{AFV}_{j}$ of a WMN $i$.

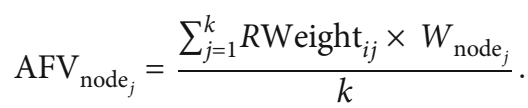

The total WSN-IoT clusters designed are $k$ solutions, $R$ Weight $_{i j}$ is the relationship weight of Node $_{i}$ and cluster $j$, when the WMN $i$ is allotted to the cluster $j$, and the value of $R$ Weight ${ }_{i j}$ will be 1 or 0 otherwise.

Once the entire FVs are calculated, the likelihood of choice $P_{\text {node }_{i}}$ in lieu of every single $\mathrm{CH}$ can be calculated using:

$$
P_{\text {node }_{i}}=\frac{W_{\text {node }_{i}}}{\sum_{j=1}^{k} W_{\text {node }_{j}}}
$$

where $k$ is equivalent to aggregate $\mathrm{CHs}$ and $W_{\text {node }_{j}}$ is the $\mathrm{WF}$ of $\mathrm{WMN} j$; local search is applied for optimal $\mathrm{CH}$ set in the region of node $_{j}$ as:

$$
\operatorname{node}_{j}(x+1)=\operatorname{node}_{j}(x)+\alpha_{i j} \times z .
$$

$\alpha_{\mathrm{ij}}$ is the association of WMN $i$ with cluster $j$, and $z$ is the randomized variable accepting value in the range $[-1,1]$ to calculate the FV by equation (11). Touching the outer range of the target WMN is not permitted in the next inhabitants.

The WMNs are assigned to the $\mathrm{CH}$-set vector on the basis of the FF described in equation (11). The neighbor WMNs join the nearby $\mathrm{CH}$ s to configure clusters. After the cluster formation, many WMNs change their state to sleep mode where other nodes in the cluster do schedule wake up to save energy. Consequently, periodic alterations will occur in the network topology. The objective of this research is finding a $\mathrm{CH}$-set as early as possible once memeWSN encounters modifications in topology.

\section{Memetic Algorithm for WSN Cluster Formation: memeWSN}

The proposed MemA-based clustering known as memeWSN for WSN-IoT is demonstrated in this section. The $\mathrm{CH}$-set is represented by a chromosome and is initially selected randomly. The $\mathrm{CH}$-set is checked by a FF to get more optimum results. Thus, the proper individuals are elected for the succeeding peers to generate a newfangled solution. The WMNs for the reconstruction of chromosomes ( $\mathrm{CH}$-set) are nominated from the populace analogous to the conventional genetic algorithm (GA). Subsequently, the choice of binary maternities in lieu of new-fangled populace, crossover in addition to the mutation will be used to make novel offsprings. The novel populace can be enhanced by calling a function designed for local search. In MemA, the indigenous search is applied to proficiently discover a less optimal solution and continue for global optimal.

The proposed memeWSN initiates the aforementioned job through computing each WMN WFs. WMNs having greater weights are designated for the early populace. The FVs of the populace are computed, and WMNs to turn into the $\mathrm{CHs}$ are designated. Furthermore, the possibility of WMN election is considered, and local search is used. In the same way, the FV of WMN to stay alive in the populace is computed. The notations used in Algorithm 1 are shown and defined in Table 1. The procedure for the formation of stable clusters using the evolutionary MemA is presented in Algorithm 1.

4.1. Genetic Representation. The classical evolutionary schemes for the example GA fails to explore numerous results of the domain area due to its inborn feature of early convergence. A MemA applies local search to reach its last stop deprived of preventing local maxima. The main stair in memA is coding the chromosome.

The set of cluster heads $S_{\mathrm{CH} i}$ of WMNs are erratically nominated from all the WSN-IoT as CHs where $i=1,2, \cdots$, $k$. Every elucidation to the issue is the set of $S_{\mathrm{CH} i}$ obtained throughout the selection of $\mathrm{CH}$ nodes. In this way, the cluster headset produced using a random permutation of WMN-IDs and the chromosome can be represented by a random set of cluster head IDs. A chromosome is the combination of WMN-IDs without repetition. Furthermore, the gene of a chromosome can be represented by a single node ID. In a network of 8 WMNs, their IDs in the WSN-IoT would be in the range 1 to 8 . In such a situation, the chromosome will represent a random combination of WMN-IDs, e.g., 54673128. Once the process of the chromosome is completed, the subsequent job is to extract $\mathrm{CH}$-set. The new nodes/genes are replaced/added to the cluster headset (chromosome) based on their weights. At each step of adding WMNs to $\mathrm{CH}$-set, the weights of $\mathrm{CHs}$ will update. The WMN bearing greater weight is substituted using lower weight WMN. The discarded node from the cluster headset is no more considered to be a $\mathrm{CH}$ in the existing round. Subsequent to encoding, the fitness of the solution is judged using a fitness function. 


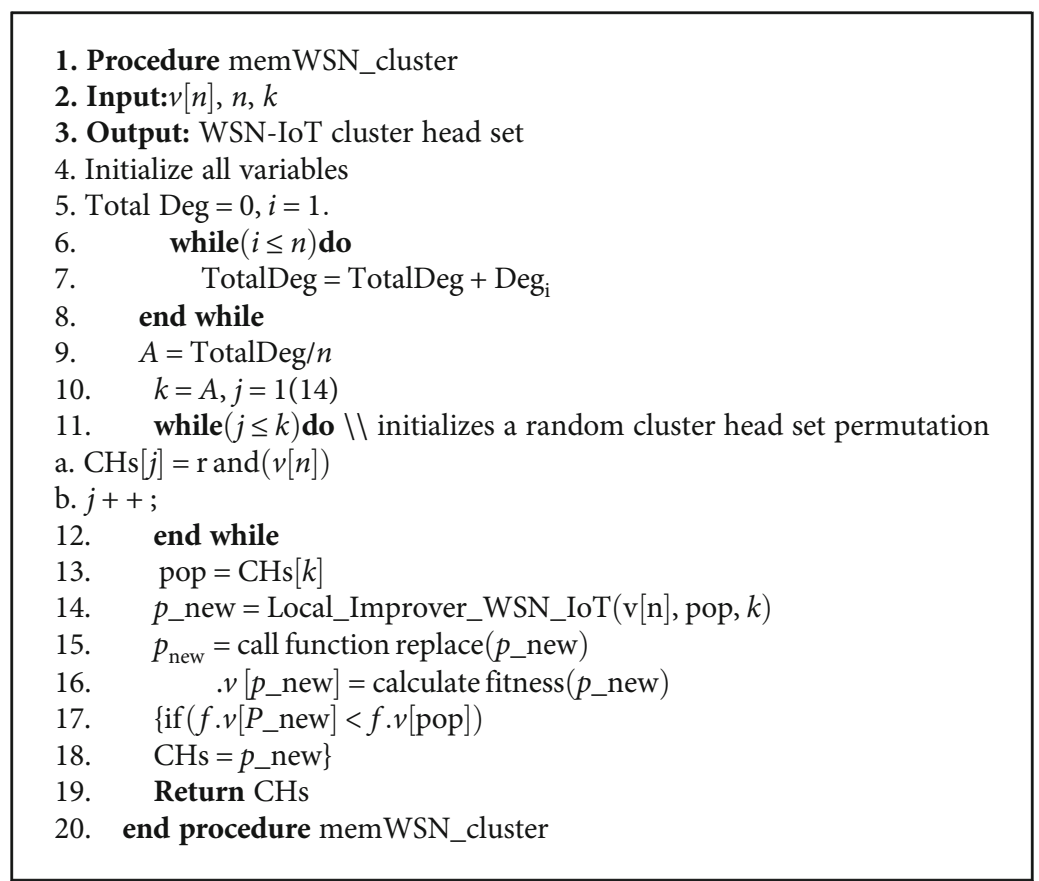

Algorithm 1: Psuedo code of memeWSN.

Table 1: Notations in Algorithm 1.

\begin{tabular}{lc}
\hline Symbol & Definition \\
\hline New $-\mathrm{CHs}$ & New CHs \\
p_new & New population \\
pop & Population \\
Deg $i$ & WMN $i$ degree \\
$f v$ & FV of $\mathrm{CH}$-set \\
$v[n]$ & WMN ID array \\
$T D$ & Total of WMN degree \\
$A$ & Average of WMN degree \\
$k$ & Total clusters \\
$m$ & Total WMNs \\
\hline
\end{tabular}

4.1.1. Objective Function. Manipulating the FVs of WMNs considered for the $\mathrm{CH}$ role in the current round using standard deviation can tell us about the quality of a chromosome. The lower the FV, the best is the solution. The FV of a $\mathrm{CH}$-set will be computed as:

$$
F(\mathrm{chr})=\sum_{i=1}^{n} \sum_{j=1}^{k} R \mathrm{Weight}_{i j}\left(W_{\text {node }_{i}}-\mathrm{AFV}_{j}\right)^{2} .
$$

In our research, the main objective is to find the set of cluster head nodes in such a way to balance clusters by assigning the cluster head role to those nodes having the max degree, low mobility, and away from other cluster heads. The selection of chromosomes for the next population is carried out after the fitness evaluation. With decent choice, better quality children are conceded to the succeeding inhab- itants for reproduction. The chromosome is nominated based on its fitness value. In this research, the mode of selection adopted is a pairwise tournament where nomination will happen without substitution. The tournament size (TS) is fixed, i.e., $\mathrm{TS}=02$. To prevent premature convergence, a local search procedure is called which mines better quality chromosomes from the population as in Algorithm 2.

4.1.2. Local Improver. In this subsection, an indigenous search is used during the selection and evaluation of a chromosome. The weights of the genes in the population are calculated. The genes with greater weights are explored in the residents. The population is examined for the genes having greater weights and swapped with low-weight genes. Algorithm 2 improves the solution locally.

The significant memetic operators are crossover (which is the offspring generated from two predecessor chromosomes) and mutation (which is children produced from one chromosome by altering a gene). The features of the newly created chromosomes are inherited from all fragments of her maternities. The newly created chromosomes are located in the populace once crossover and mutation are performed. The chromosomes with good features are swapped by low-quality chromosomes.

The newly created inhabitants can be used for the additional execution of the procedure. The procedure is repeated till the ending criterion hits. The top solution is resumed after the final repetition of the memeWSN procedure.

\section{Experiment Evaluation}

To evaluate the efficiency of the proposed memeWSN clustering scheme, numerous simulation experimentations have been carried out in EstiNet 9.0. In a $1000 \mathrm{~m} \times 1000 \mathrm{~m}$ square 


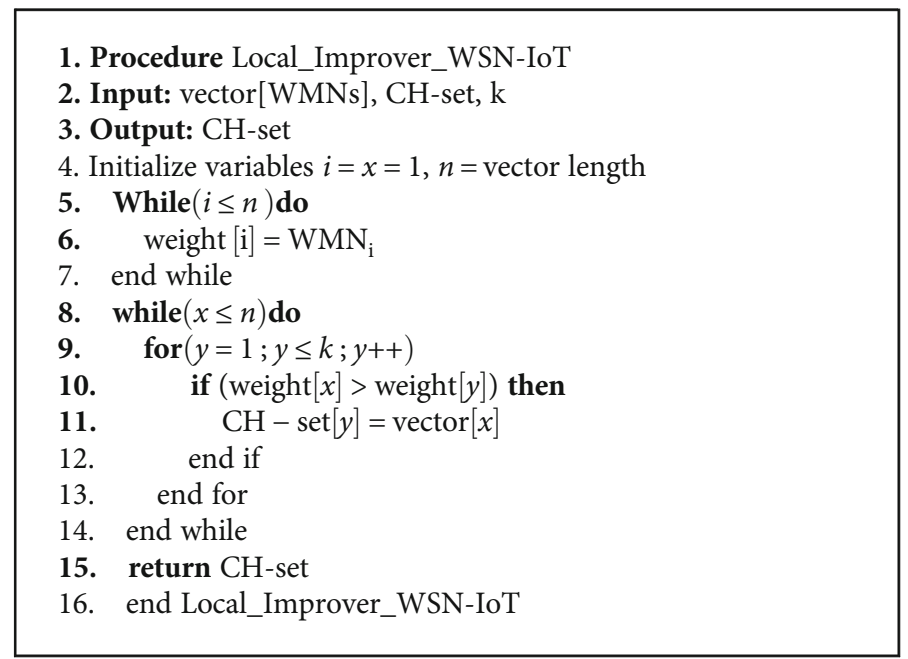

Algorithm 2: Pseudocode of procedure local-improver for WSN-IoT.

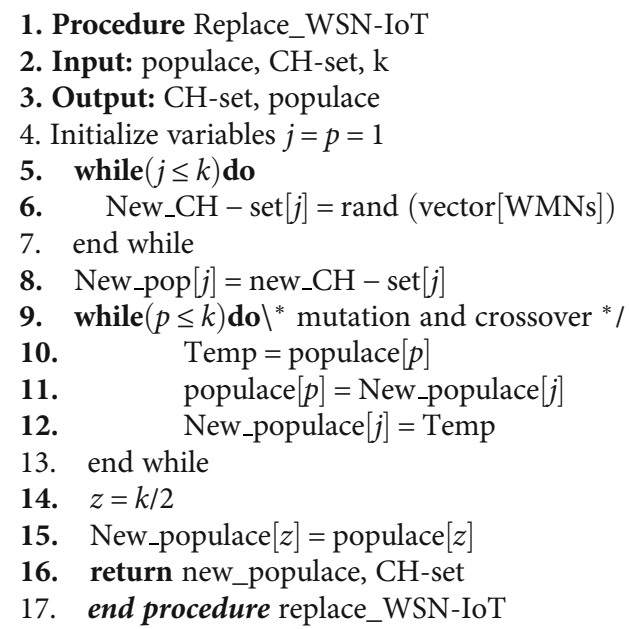

Algorithm 3: Pseudo code of procedure-replace for WSN-IoT.

simulation area, the WMNs (up to 500) are deployed in a random way. The simulation parameters are adopted from [17]. The speed of WMNs varies from $1 \mathrm{~km} / \mathrm{h}$ to $80 \mathrm{~km} / \mathrm{h}$. Every WMN is equipped with a default omnidirectional antenna. The broadcast range of every antenna varies in the range between $100 \mathrm{~m}$ and $300 \mathrm{~m}$. Each WMN has a storage (queue) for outgoing and incoming data and maintains the mobility statistics of their neighbors. The traffic sources are generated by a continuous bit rate mechanism. The maximum limit for the generation of traffic is set to twenty (20) packets/s. The simulations are executed for fifty (50) minutes. The average values of a hundred simulation rounds are demonstrated in the form of graphs. Table 2 shows the parameters used during the simulation as in [17].

The performance of the memeWSN algorithm is compared with PBC-CP [16] and EPSO-C [17] for the performance measurement of control overhead and computation. Similarly, MobAC [15] for the performance evaluation of cluster lifetime and reaffiliation. To assess the efficiency of the proposed memeWSN, the metrics discussed below are measured.

The number of clusters (NoC) or cluster count (CC): The WMNs are distributed to several simulated groups known as clusters in cluster-based WSN-IoTs. Here, the NoC or CC denotes the sum of virtual sets gained when executing the cluster foundation procedure. The fewer NoCs represent the stability of WSN-IoT clusters. The issues for instance channel access scheduling, reusing frequency, energy depletion, and latency may arise when the NoCs may increase

Re-affiliation rate $(R R)$ : The affiliation and deaffiliation of WMNs as $\mathrm{CH}$ or member throughout a certain interval of time represent reaffiliation rate. In cluster-based routing, a WMN links to the adjacent $\mathrm{CH}$ and vacates the former. A WMN may reaffiliate once its $\mathrm{CH}$ no more survives, and alternative WMN performs the role of a $\mathrm{CH}$ in the vicinity. The reaffiliation may also happen once the $\mathrm{CH}$ is not within the broadcast range of a WMN. The cluster lifetime will decrease when $\mathrm{RR}$ is high and vice versa

Control overhead (CO): The number of packet exchanges to maintain the statistics of topology variations denotes the CO. Several control message (CM) exchanges happen throughout the cluster construction stage. To measure the $\mathrm{CO}$ metric, the CMs received/sent at some interval are computed

5.1. NoC or CC. Several tests have been carried out to compute the CC metric once we increase the number of nodes from 50 to 500 . The incremental step 50 is adopted to increase WMNs in individual simulation experiments. The random waypoint mobility model is practiced in this set of experiments. The speed of WMNs varies in the range 1 kilometer/h to 80 kilometers/h, i.e., 1 kilometer/hour to 5 kilometers/hour (walking), 5 kilometers/hour to 20 kilometers/hour (running), and 20 kilometers/hour to 80 kilometers/hour (vehicle) drive. Similarly, the transmission range is 100-200 meters for various experiments. To measure the CC metric, the outcomes derived during 
TABLE 2: Simulation parameters.

\begin{tabular}{lccc}
\hline Parameters & Value & Parameters & Value \\
\hline 3d beam width & 360 degrees & Simulation time & $50 \mathrm{~min}$ \\
Pointing direction & 90 degrees & Max $(x)$ & Max $(y)$ \\
Angular speed & 0 degrees/sec & Node space & $1000 \mathrm{~m}$ \\
Link bandwidth & $11 \mathrm{Mbps}$ & Number of nodes & $1000 \mathrm{~m}$ \\
Frequency channel & 3 & Data packet size & $50-500$ \\
Bit error rate & 0.0 & Mobility speed & $1200 \mathrm{bytes}$ \\
Frequency (MHz) & 2400 & Shadowing standard deviation & $1,5,10 \mathrm{~m} / \mathrm{sec}$ \\
Transmission power & $15 \mathrm{dbm}$ & Close in reference distance & 4.0 \\
Carrier sense threshold & $57 \mathrm{dbm}$ & System loss & $1.0 \mathrm{~m}$ \\
Antenna gain & $1.0 \mathrm{dbi}$ & Antenna height & 1.0 \\
Fading variation & 10.0 & Ricean factor $K$ & $1.5 \mathrm{~m}$ \\
Average building height & $10 \mathrm{~m}$ & Max queue length & $10.0 \mathrm{db}$ \\
Street width & $30 \mathrm{~m}$ & RTS threshold & $50 \mathrm{pkts}$ \\
Path loss exponent & 2.0 & $3000 \mathrm{bytes}$ \\
\hline
\end{tabular}

the simulation are presented in the form of a graph in Figures 1 and 2. The curves presented in Figures 1 and 2 show the NoCs for different size networks (i.e., up to $500 \mathrm{WMNs}$ ). The NoCs will increase with the increase in the number of WMNs as presented in Figure 1. The graph shows that memeWSN forms less number of clusters as compared to MobAC, PBC-CP, and EPSO-C. In memeWSN, the NoCs is computed based on node neighbors. The average degrees of WMNs are identified to compute the NoCs. Hence, the memeWSN has less NoCs, and the steadiness of clusters may also be ensured if node degree is in consideration. After memeWSN, PBC-CP performs glowing related to other protocols as presented in Figure 1. Analysing the curves demonstrated in Figure 1, we notice that EPSO-C performs pitiably, and the NoCs generated by MobAC is significantly fewer compared to EPSOC. MobAC is emphasized on the steadiness of clusters by guessing the expected movement of WMNs throughout cluster creation. EPSO-C focuses on the optimum computations involved in the cluster creation process. The outcomes confirm that memeWSN leaves behind state-of-the-art cluster-based routing protocols w.r.t CC. PBC-CP performs well after memeWSN. To evaluate the performance of memeWSN in more detail, a sequence of simulation trials are conducted for another broadcast range, i.e., 200 meters. The outcomes of the succeeding trials are depicted in Figure 2. The arcs in Figure 2 indicate that the wireless broadcast ranges of WMNs affect the efficiency of cluster-based routing schemes. The CC metric drops greatly with high broadcast ranges. With a high broadcast range, the $\mathrm{CH}$ will cover a big region and the number of WMNs $\mathrm{CH}$ serve may grow hence results in less NoCs. The magnitude of backbone WSN-IoT will reduce when we rise the wireless broadcast span of WMNs. All protocols perform the same way as in Figure 1.

5.2. Cluster Lifetime. The effect of node speed on the lifetime of $\mathrm{CHs}$ is evaluated in this section. The WMNs with high motion may reduce the lifespan of $\mathrm{CHs}$, deploying
100 WMNs in WSN-IoT to execute the simulation. The transmission range of each WMN is fixed to two hundred in the main experiment. The WMNs are moving at a speed varying from 1 kilometer/hour to 80 kilometers/hour where the mobility characteristics are adopted as in [17]. As shown in Figure 3, the proposed memeWSN clustering algorithm selects $\mathrm{CH}$ s for an extended period. Steady clusters are designed via a memeWSN clustering scheme because the comparative motion of WMNs and their neighbors is assumed for the duration of $\mathrm{CH}$ s choice. The proposed memeWSN outperforms PBC-CP, MobAC, and EPSO-C in terms of cluster steadiness. MobAC performs fine after memeWSN since it takes the future motion of WMNs throughout the cluster creation. The behavior of WMNs may not project genuinely in long term. In memeWSN, the cluster partners stay connected to the $\mathrm{CH}$ for an extended period. The comparative motion of WMNs is dignified throughout the CHs election, and a WMN with a higher degree and comparative movement is the top contender to be a $\mathrm{CH}$.

The evaluation of bars presented in Figure 3 demonstrate that memeWSN create steady and long life clusters compared to MobAC. EPSO-C and PBC-CP choose unsteady CHs. The EPSO-C is better as compared to PBC-CP. Thus, PBC-CP selects unsteady $\mathrm{CHs}$ once the speed of WMNs increases. The movement of nodes is not taken into consideration throughout the cluster formation process. The wireless broadcast range of WMNs is amplified to 300 meters in the next test, and the outcomes are exposed in Figure 4. The chart demonstrates that the wireless broadcast range of WMNs influences considerably the efficiency of all clustering schemes assumed in this experiment comprising our recommended memeWSN scheme. More steady clusters are obtained when we increase the broadcast range of WMNs. The lifetime of the cluster and network may increase. The suggested memeWSN performs fine once we increase the transmission range. With more broadcast range, the WMNs reaffiliation drops and a $\mathrm{CH}$ will handle large-size WSN-IoT. 


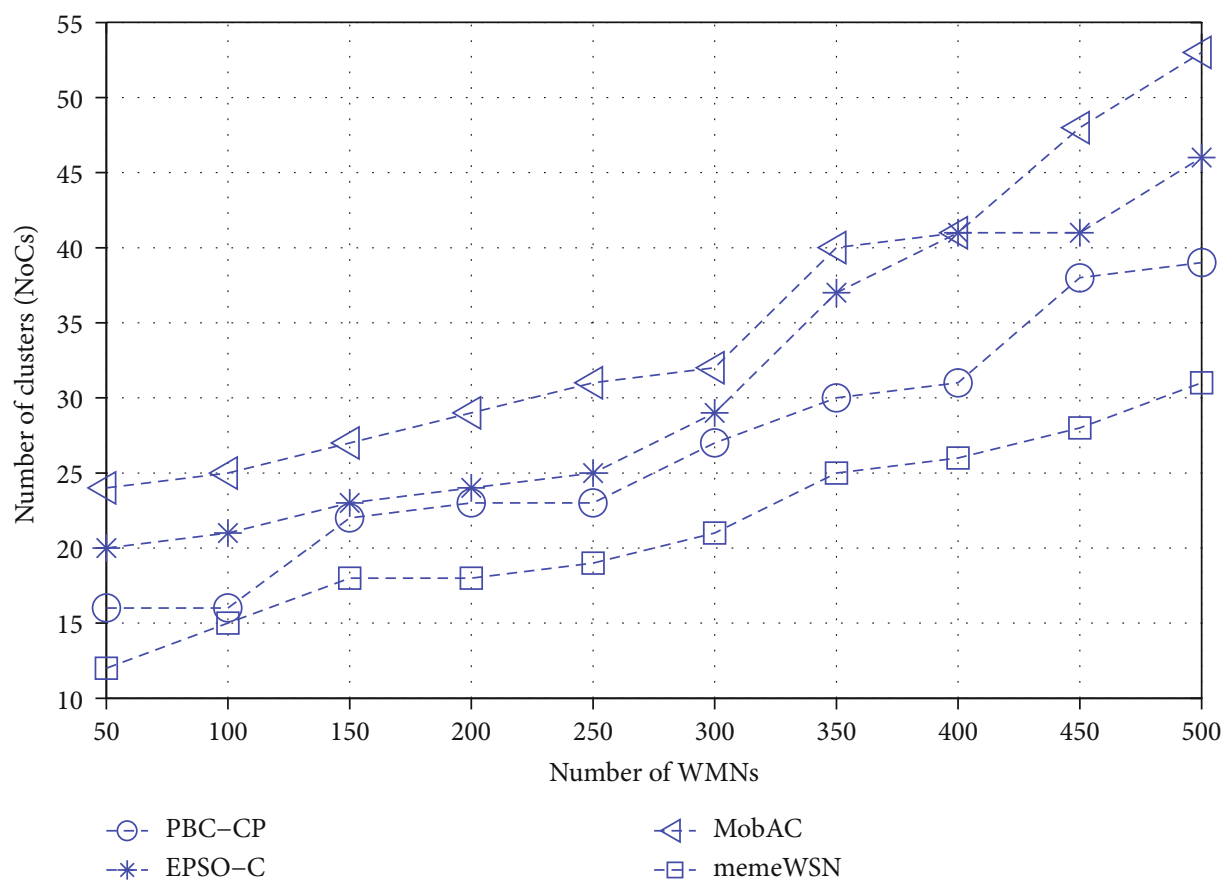

FIgUre 1: Average clusters vs. WSN-IoT size (WMN broadcast range $100 \mathrm{~m}$ ).

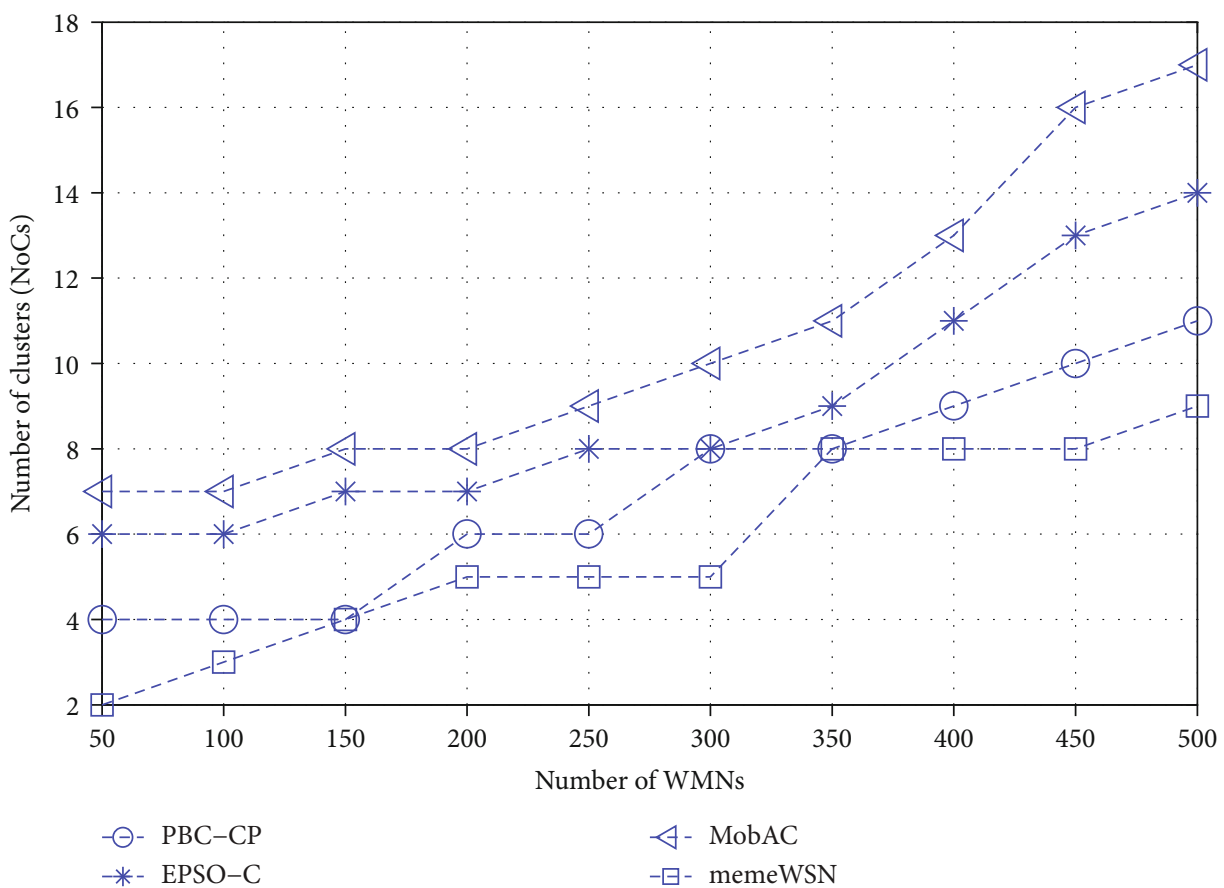

FIgURe 2: Average clusters vs. WSN-IoT size (WMN broadcast range $200 \mathrm{~m}$ ).

5.3. Reaffiliation Rate (RR). To assess the efficiency of clustering schemes w.r.t reaffiliation rate for different WMN speed and wireless broadcast range, a series of simulation tests have been carried out in Figures 5 and 6. Reaffiliation may occur if a WMN leaves its present $\mathrm{CH}$ and join an alternative cluster or a $\mathrm{CH}$ move beyond the radio broadcast range of a member $\mathrm{WMN}$. The WMN is unable to stay linked to its $\mathrm{CH}$ to any further extent. With the rise in the speed of WMNs, the reaf- filiation is more often as WMNs leave their $\mathrm{CHs}$ more frequently. The radio broadcast range of all nodes is fixed to 200 meters. A total of 100 nodes are deployed randomly in a $1000 \mathrm{~m} \times 1000 \mathrm{~m}$ simulation area initially.

The nodes are moving at a speed between 1 kilometer/hour to 80 kilometers/hour. The outcomes are averaged for different experiments. The average reaffiliation rate for more than 100 dissimilar runs is presented in Figure 5. As depicted 


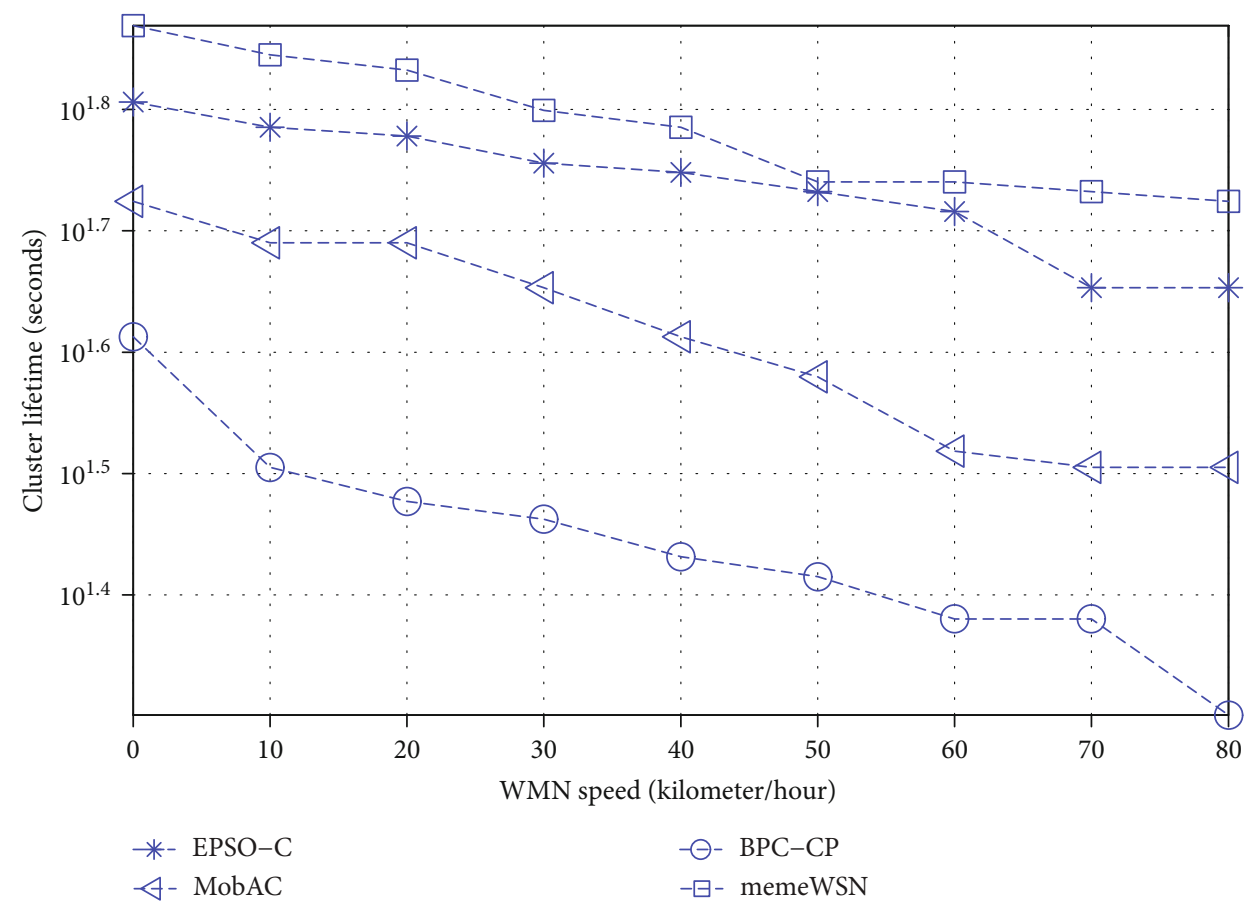

Figure 3: WMNs speed vs. cluster lifetime (WMN broadcast range $200 \mathrm{~m}$ ).

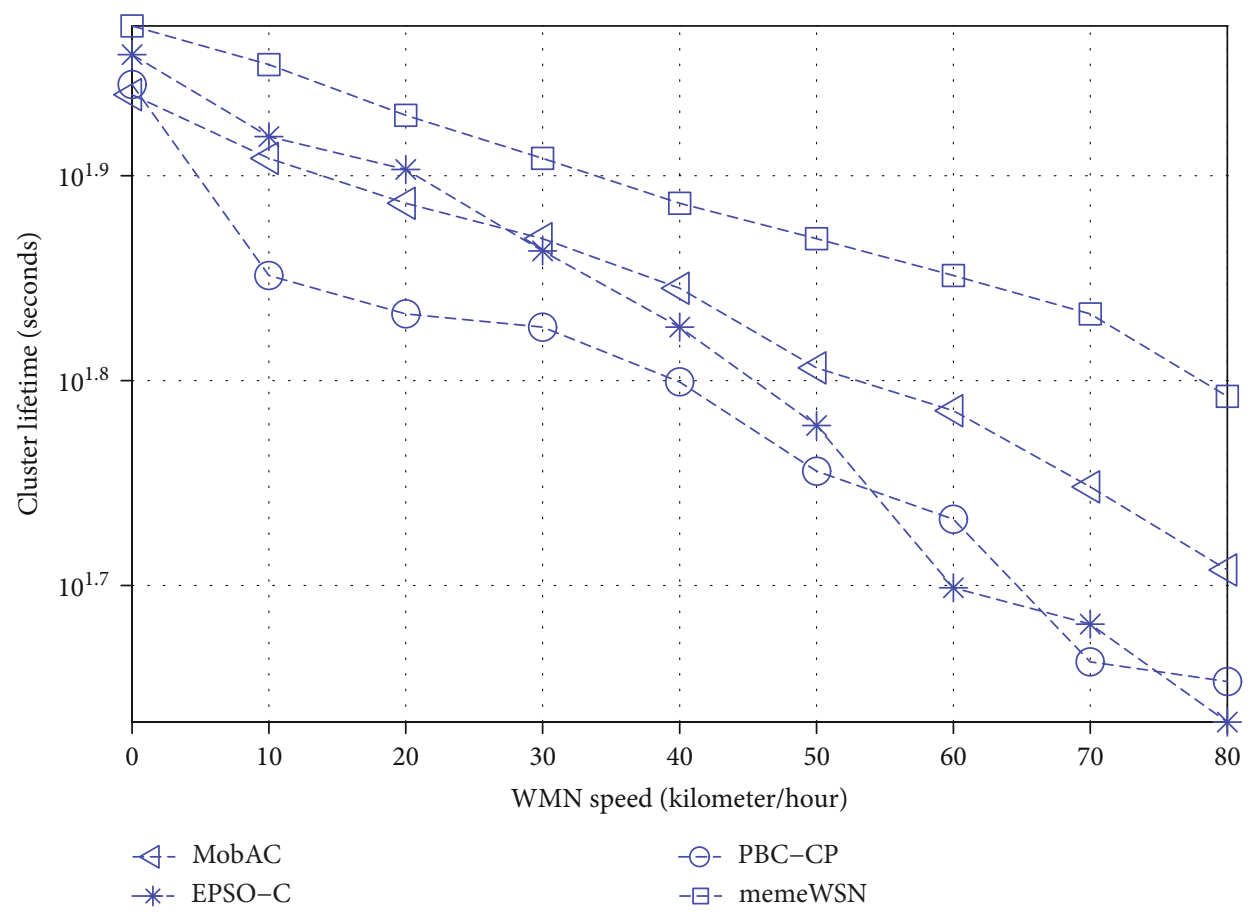

FIgURE 4: WMNs speed vs. cluster lifetime (WMN broadcast range $300 \mathrm{~m}$ ).

in the graph, the rise in reaffiliation rate is noted in memeWSN, MobAC, PBC-CP, and EPSO-C when increasing the speed of WMNs. The efficiency of memeWSN is well as presented in the arcs because it has the lowermost RR as equated to other protocols under consideration. The lifetime of $\mathrm{CHs}$ is lengthy once the memeWSN cluster formation procedure is executed, and it indicates that the reaffiliation will be low as reclustering mechanism is initiated less repeatedly when the steady $\mathrm{CH}$ s are nominated. This is because the $\mathrm{CHs}$ are selected on the basis of WMN remaining energy, degree, and relative mobility. Therefore, the $\mathrm{CHs}$ lifetime will increase and the participants vacate the present cluster less frequently. The efficiency of MobAC is improved after memeWSN, since in MobAC, the WMN future mobility is taken 


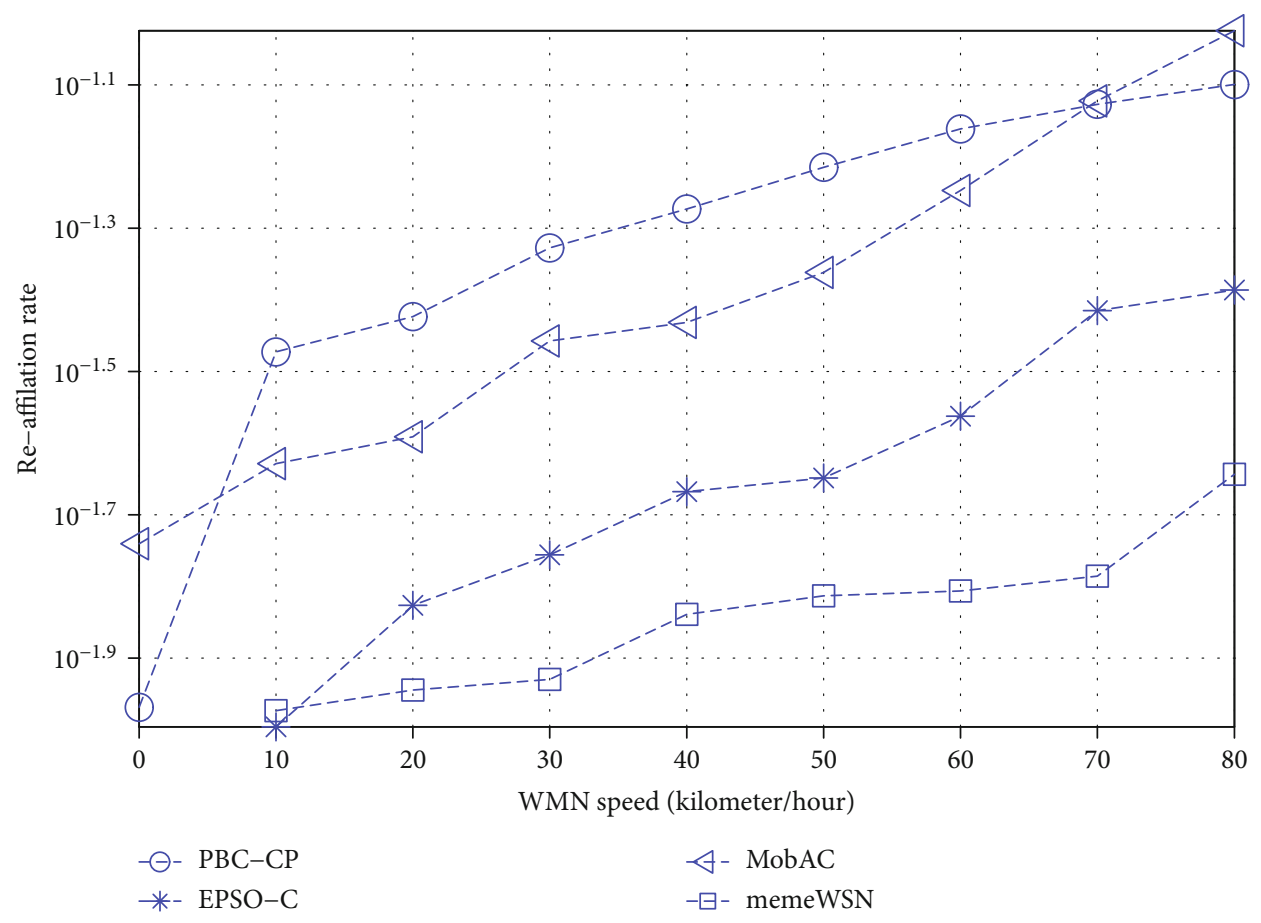

FIgURE 5: Reaffiliation rate vs. WMN speed.

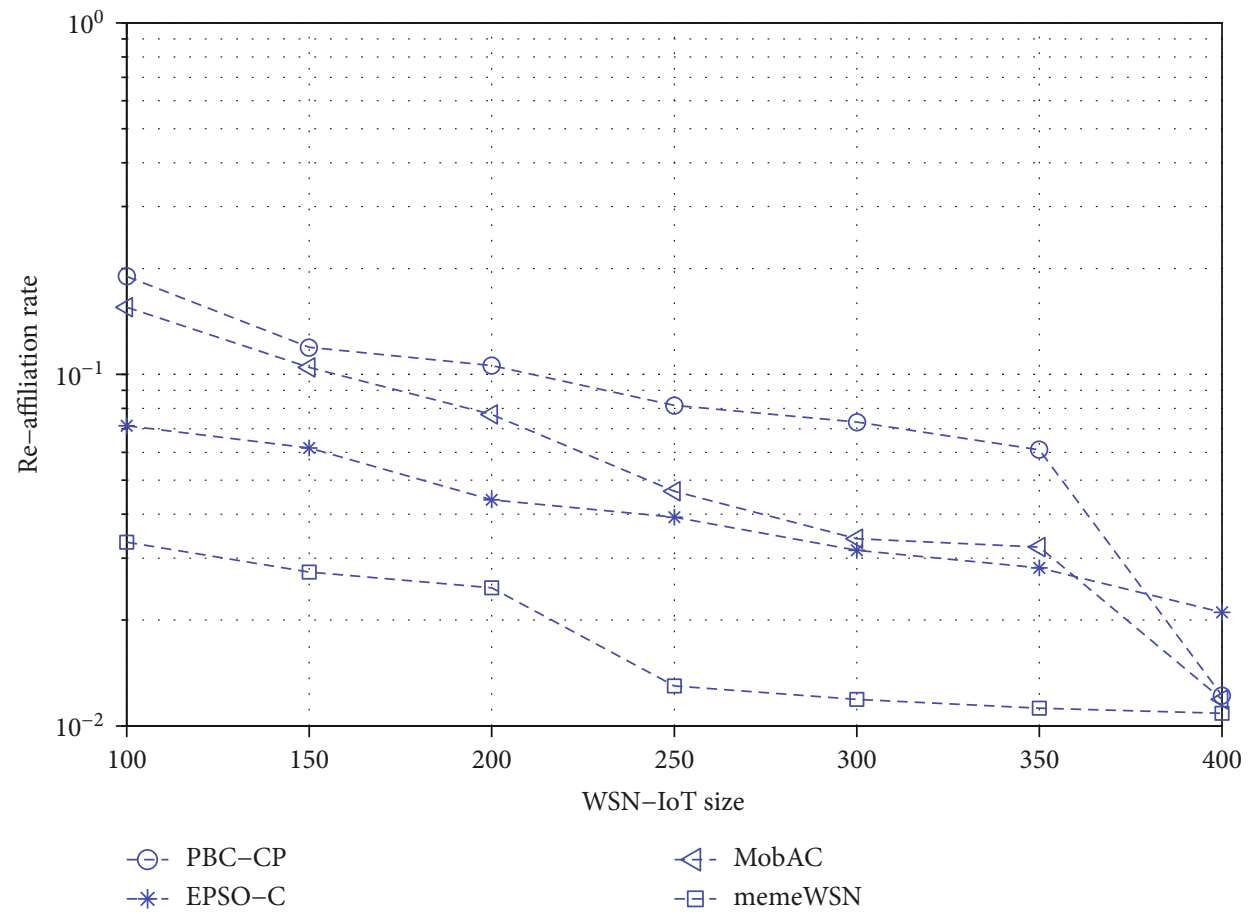

FIGURE 6: Reaffiliation rate vs. WMN wireless broadcast range.

into consideration throughout the $\mathrm{CH}$ election process. The efficiency of MobAC is better among all state of the art clustering algorithm under consideration because the neighbors of the selected $\mathrm{CH}$ s remain for a long time, and hence, the probability of reaffiliation decreases. The worst performance w.r.t reaffiliation is observed in $\mathrm{PBC}-\mathrm{CP}$ compared to
MobAC and EPSO-C. The mobility of nodes is not considered in $\mathrm{PBC}-\mathrm{CP}$ during the cluster formation procedure. Hence, unstable clusters may result. The same simulation experimentations are repeated for a different size network, i.e., $50 \mathrm{WMNs}$ to $500 \mathrm{WMNs}$. The results are presented in the form of a graph in Figure 6. Incremental step 50 was used 


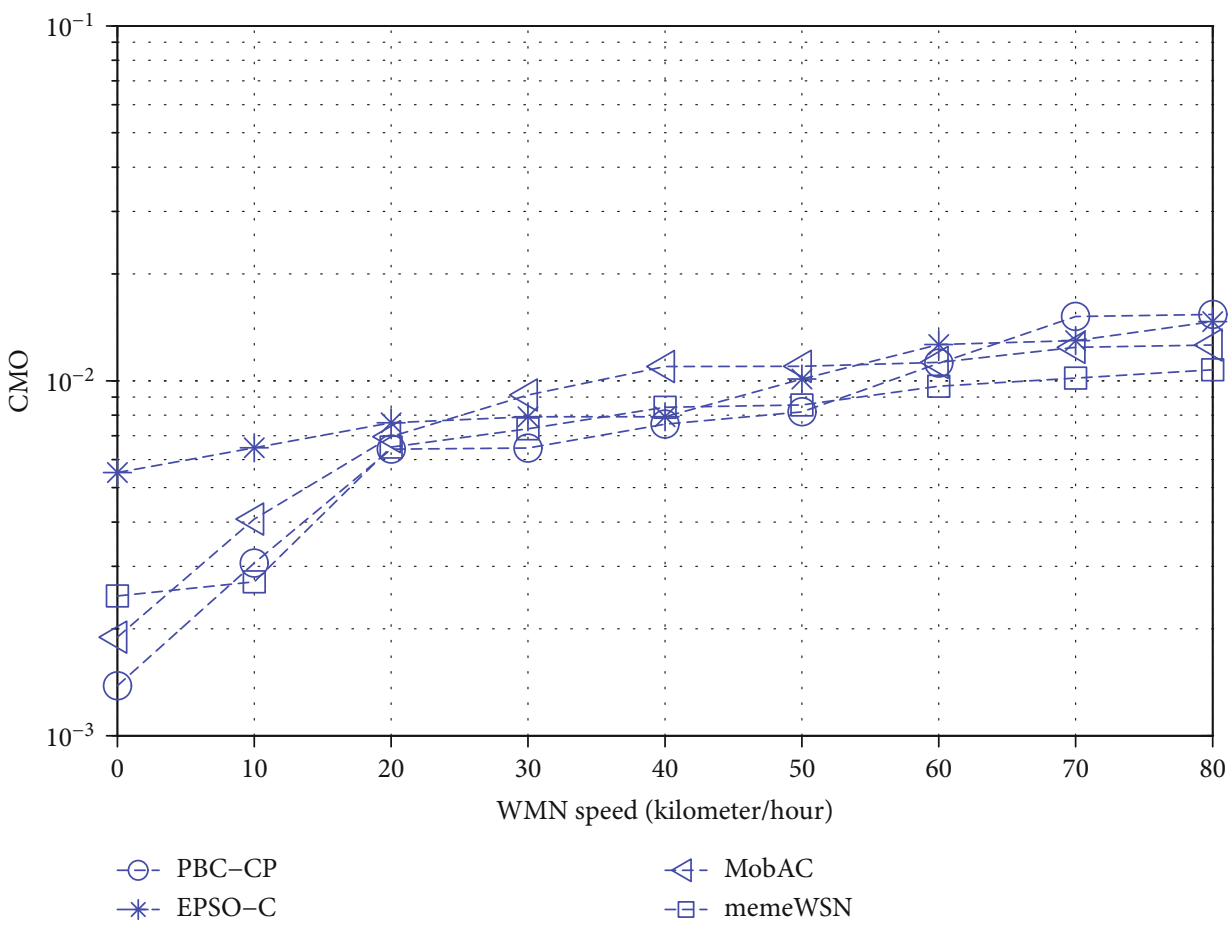

FIgURE 7: WSN-IoT WMN speed vs. CMO (broadcast range 200 meters).

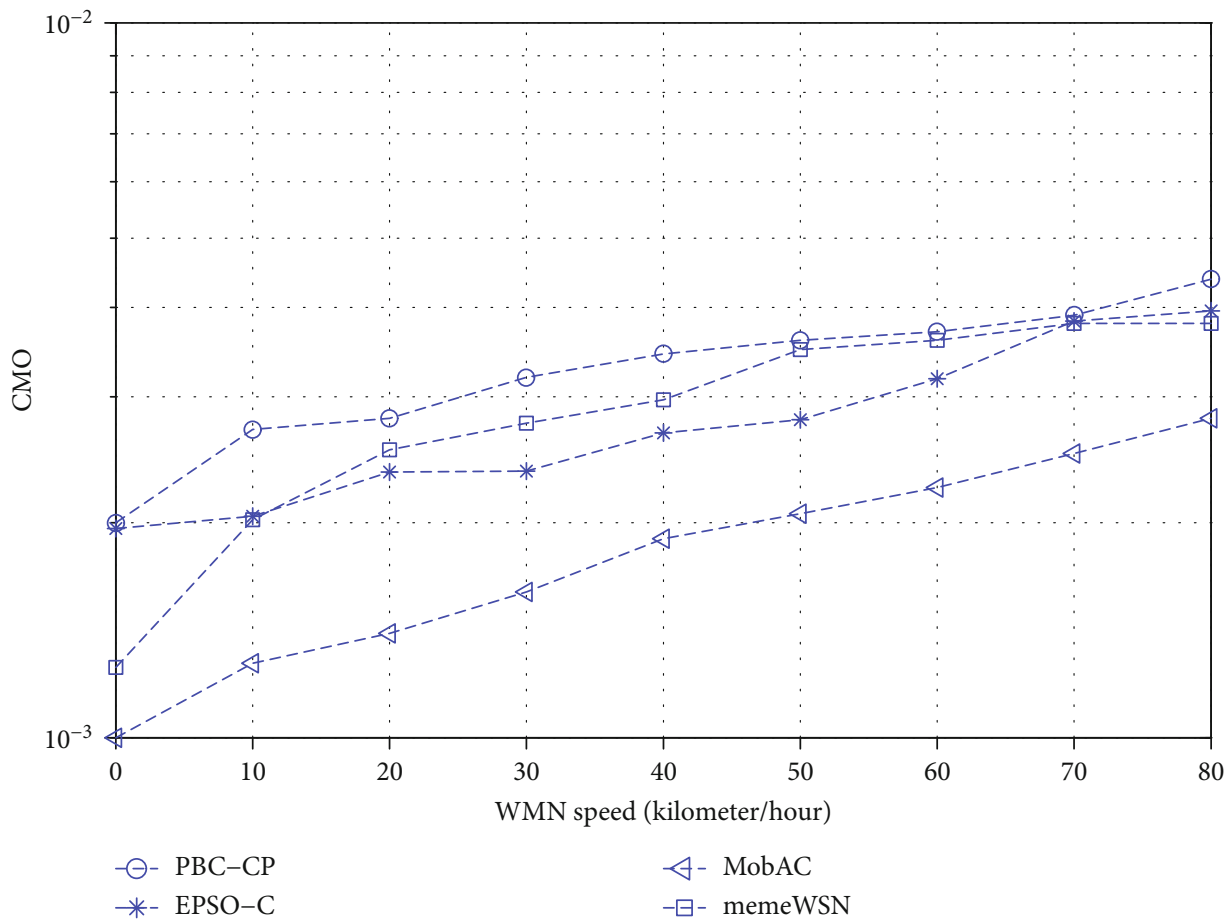

Figure 8: WSN-IoT WMN speed vs. CMO (broadcast range $300 \mathrm{~m}$ ).

to evaluate the performance of memeWSN with a diverse number of WMNs in WSN-IoT. The graph presented in Figure 6 shows the decrease in reaffiliation rate when the WSN-IoT size becomes large. In a large-scale network, the $\mathrm{CHs}$ serve a large number of nodes, and the changes in topology are communicated less frequently. The simulation area was the same as in the previous experiment. The curve at the bottom of the diagram in Figure 6 shows the decrease in memeWSN reaffiliation rate when the size of the network increases in line with other cluster-based routing algorithms. In memeWSN, the CHs are selected on the basis of relative mobility, remaining energy, and node degree, and steady 
clusters are achieved. The reaffiliation rate will be low when the number of steady clusters increases.

5.4. Control Message Overhead (CMO). Numerous packets exchange is the essential part throughout the $\mathrm{CH}$ selection method in WSN-IoT. In this segment, the number of messages swapped during the course of the cluster creation and maintenance stage is noted and demonstrated. The efficiency of memeWSN is matched with MobAC, EPSO$\mathrm{C}$, and $\mathrm{PBC}-\mathrm{CP}$.

In this set of simulation tests, fifty WMNs are deployed in a random way. The simulation region $1000 \mathrm{~m} \times 1000 \mathrm{~m}$ is assumed. Random waypoint mobility was adopted in the simulation tests. The radio transmission range of WMNs is fixed to 200 meters. The WMNs are moving at a speed between 0-5 kilometers/h (walk), 5-20 kilometers/hour (running), and 20-80 kilometers/hour (car). The outcomes presented in the form of a line chart is depicted in Figure 7. The experiments are conducted for another transmission range of 300 meters, and the obtained results are presented in Figure 8. As exposed in the graph, the CMO of PBC-CP is low while the WMNs are moving with a gentle speed such as no more than $40 \mathrm{~km} / \mathrm{h}$ plus becomes higher when the WMN movement is faster. GA hurts from a local maximum problem, and unsteady clusters may result once the WMN speed turns out to be high. The identical outcome can be seen in other protocols under consideration comprising memeWSN as the reclustering method is initiated frequently when the mobility is high. The CMO may decrease once we rise the radio broadcast range of WMNs. The high transmission range may drink extra energy throughout the WSN-IoT processes. The increase in WSN-IoT lifetime may not guarantee when the broadcast range of WMNs is high. More communication is required when finding optimal $\mathrm{CHs}$, and the optimization techniques have great $\mathrm{CMO}$ values as shown in Figures 7 and 8. EPSO-C and MobAC offer consistent performance when the radio range of WMNs is 200 meters. When the broadcast range is high, EPSO-C and MobAC have the lowest CMO. Our proposed memeWSN algorithm requires more message exchanges for the duration of cluster creation. The parameters like WMN degree, its energy, and relative mobility are taken into consideration, and the accurate calculation may proliferate the number of control messages. The optimal CHs are computed on the cost of more overhead, but when the optimum CHs are identified, the reclustering method will initiate less frequently.

\section{Conclusion and Future Work}

In wireless sensor network-enabled Internet of Things, the resource limitation requires a handsome algorithm for cluster formation to route data with little resources in order to increase the lifetime of WSN-IoT. Optimization methods, for example, evolutionary algorithms, PSO, and linear programming, may be used to form stable and long life clusters. The stable and balanced clusters may be obtained when we consider parameters such as mobility, degree, and energy of WMNs. In this paper, the $\mathrm{CH}$ selection method is optimized using an evolutionary memetic algorithm. A minimization function is modeled to check the fitness of a solution. The $\mathrm{CH}$-set represents a chromosome in the proposed scheme. The memetic algorithm has a built-in searching mechanism that prevents it from premature convergence. The proposed algorithm is validated through a series of simulation experiments. The simulation experiments demonstrate that the proposed algorithm outperforms the state-of-the-art clustering schemes in WSN.

In the future, a modified version of the memetic algorithm can be used to form balanced clusters in high-speed networks such as vehicular ad hoc networks and flying ad hoc networks, i.e., unmanned aerial vehicles.

\section{Data Availability}

Regarding data availability, no data were used to support this study. We discussed the results based on simulation which is already discussed in the paper in detail.

\section{Conflicts of Interest}

The authors declare that they have no conflicts of interest.

\section{Acknowledgments}

This work was supported by the Zayed University RIF grant activity code R20129.

\section{References}

[1] P. Gupta and P. R. Kumar, "The capacity of wireless networks," IEEE Transactions on Information Theory, vol. 46, no. 2, pp. 388-404, 2000.

[2] E. M. Belding-Royer, "Hierarchical routing in ad-hoc mobile networks," Wireless Communications and Mobile Computing, vol. 2, no. 5, p. 532, 2002.

[3] C. E. Perkins, Ad-hoc Networking, Addison-Wesley, 2001.

[4] S. Basagni and I. Chlamtac, "A generalized clustering algorithm for peer-to-peer networks," in Workshop on Algorithmic Aspects of Communication, Bologna, Italy, July 1997.

[5] N. Shah, S. A. Abid, D. Qian, and W. Mehmood, "A survey of $\mathrm{P} 2 \mathrm{P}$ content sharing in MANETs," Computers \& Electrical Engineering, vol. 57, pp. 55-68, 2017.

[6] A. H. Azni, R. Ahmad, K. Seman, N. H. Alwi, and Z. A. Noh, "Correlated topology control algorithm for survival network in MANETS," in Advanced Computer and Communication Engineering Technology. Lecture Notes in Electrical Engineering, vol 362, H. Sulaiman, M. Othman, M. Othman, Y. Rahim, and N. Pee, Eds., pp. 93-102, Springer, Cham, 2016.

[7] K. Deb, K. Sindhya, and J. Hakanen, "Multi-objective optimization," in Decision Sciences: Theory and Practice, pp. 145184, CRC Press, 2016.

[8] G. Kannan and T. Sree Renga Raja, "Energy efficient distributed cluster head scheduling scheme for two tiered wireless sensor network," Egyptian Informatics Journal, vol. 16, no. 2, pp. 167-174, 2015.

[9] T. M. Behera, S. K. Mohapatra, U. C. Samal, M. S. Khan, M. Daneshmand, and A. H. Gandomi, "Residual energybased cluster-head selection in WSNs for IoT application," IEEE Internet of Things Journal, vol. 6, no. 3, pp. 5132-5139, 2019. 
[10] F. Ling, "Leader based group routing in disconnected mobile ad hoc networks with group mobility," Wireless Personal Communications, vol. 71, no. 3, pp. 2003-2021, 2013.

[11] U. Venkanna and R. Leela Velusamy, "Distributed cluster head election in MANET by using AHP," Peer-to-Peer Networking and Applications, vol. 9, no. 1, pp. 159-170, 2016.

[12] C. Konstantopoulos, D. Gavalas, and G. Pantziou, "Clustering in mobile ad hoc networks through neighborhood stabilitybased mobility prediction," Computer Networks, vol. 52, pp. 1797-1824, 2008.

[13] H. Cheng, S. Yang, and J. Cao, "Dynamic genetic algorithms for the dynamic load balanced clustering problem in mobile ad hoc networks," Expert Systems with Applications, vol. 40, pp. 1381-1392, 2013.

[14] H. Ali, W. Shahzad, and F. A. Khan, "Energy-efficient clustering in mobile ad-hoc networks using multi-objective particle swarm optimization," Applied Soft Computing, vol. 12, no. 7, pp. 1913-1928, 2012.

[15] V. Singh and R. B. Lohani, "Mobility aware energy efficient clustering for wireless sensor network," in 2019 IEEE International Conference on Electrical, Computer and Communication Technologies (ICECCT), pp. 1-6, Coimbatore, India, Febuary 2019.

[16] A. Pathak, "A proficient bee colony-clustering protocol to prolong lifetime of wireless sensor networks," Journal of Computer Networks and Communications, vol. 2020, Article ID 1236187, 9 pages, 2020.

[17] C. Vimalarani, R. Subramanian, and S. N. Sivanandam, "An enhanced PSO-based clustering energy optimization algorithm for wireless sensor network," The Scientific World Journal, vol. 2016, Article ID 8658760, 11 pages, 2016.

[18] M. Ahmad, A. A. Ikram, R. Lela, I. Wahid, and R. Ulla, "Honey bee algorithm-based efficient cluster formation and optimization scheme in mobile ad hoc networks," International Journal of Distributed Sensor Networks, vol. 13, no. 6, Article ID $155014771771681,2017$. 